\title{
O TORMENTO DA HUMANIDADE: REQUERENTES DE ASILO LGBT NA EUROPA ENFRENTANDO OS LIMITES DOS DIREITOS HUMANOS
}

\section{THE ORDEAL FOR HUMANITY: LGBTI ASYLUM SEEKERS IN EUROPE FACING THE LIMITS OF HUMAN RIGHTS}

\author{
Lorenzo Bernini ${ }^{1}$ \\ Tradução: Gustavo Bussmann ${ }^{2}$ Ferreira e Katya Kozicki ${ }^{3}$
}

\begin{abstract}
Como citar este artigo / How to cite this article (informe a data atual de acesso / inform the current date of access):
BERNINI, Lorenzo. O tormento da humanidade: requerentes de asilo LGBT na Europa enfrentando os limites dos direitos humanos. Tradução Gustavo Bussmann Ferreira e Katya Kozicki. Revista da Faculdade de Direito UFPR, Curitiba, PR, Brasil, v. 63, n. 2, p. 217-228, ago. 2018. ISSN 2236-7284. Disponível em: <https://revistas.ufpr.br/direito/article/view/60556>. Acesso $\quad$ em: $30 \quad$ ago. $2018 . \quad$ DOI:
\end{abstract} http://dx.doi.org/10.5380/rfdufpr.v63i2.60556.

\section{RESUMO}

O que é o "humano" no humano? O que faz um ser vivente ser humano? Em caso de dúvida, quem decide se um ser vivente é um ser humano? Uma cultura política liberal baseada no valor dos direitos humanos não pode evitar essas questões. Assim, este artigo usará diferentes quadros interpretativos (Thomas Hobbes, Michel Foucault, Leo Bersani, Giorgio Agamben, Judith Butler, Lee Edelman, Jasbir Puar) para dar conta da permanência de uma decisão soberana sobre o humano no governo biopolítico do presente. Exemplo disso é condição de lésbicas, gays, bissexuais, transgêneros e intersexuais vindos do continente africano para requerer asilo na Europa. Perseguidos em seus países por sua orientação sexual ou identidade de gênero, em muitos casos atravessam o Mediterrâneo em barcos precários até a terra firme - reviravolta do destino - nas mesmas ilhas em que o fascismo italiano costumava confinar homens homossexuais. Lá, são "recebidos” em campos para imigrantes ilegais onde sua completa humanidade, negada por seus países de origem, será examinada por uma comissão. Apenas se reconhecidos como membros autênticos de uma minoria sexual eles se beneficiarão da totalidade dos direitos humanos no continente europeu. Caso contrário, correm o risco de serem forçados a sair da Europa - e da humanidade. Longe de ser uma viagem de esperança, com origem na barbaridade para chegar-se à modernidade, a jornada, iniciada na África e para a Europa, torna-se um arcaico tormento.

\section{PALAVRAS-CHAVE}

Teoria Queer. Homonacionalismo. Leo Bersani. Lee Edelman. Jasbir Puar.

\section{ABSTRACT}

What's "human" in the human? What makes a living being a human being? In case of doubt, who decides whether a living being is a human being? A liberal political culture based on the value of human rights cannot avoid these questions. This article will use different interpretative frames

\footnotetext{
${ }^{1}$ Università degli Studi di Verona. Artigo original disponível em: <https://goo.gl/RQuHgN>.

${ }^{2}$ Mestre em Direito (2015) pela Universidade Federal do Paraná e doutorando em Direito na mesma instituição; bolsista Capes.

${ }^{3}$ Mestre e doutora em Direito. Professora titular da PUCPR e professora associada IV da UFPR; programas de graduação e pós-graduação em Direito. Pesquisadora do CNPq.
} 
(Thomas Hobbes, Michel Foucault, Leo Bersani, Giorgio Agamben, Judith Butler, Lee Edelman, Jasbir Puar) in order to give account of the permanence of a sovereign decision on the human in the biopolitical governance of the present. The condition of African lesbian, gay, bisexual, transgender, and intersex asylum seekers in Europe is exemplary of this. Persecuted in their countries for their sexual orientation or gender identity, in many cases they cross the Mediterranean on makeshift boats to land - twist of fate - on the same islands where Italian Fascism used to confine homosexual men. There they are "received" in camps for illegal immigrants where their full humanity, denied by their countries of origin, will be taken into exam by a commission. Only if recognized as authentic members of a sexual minority, they will benefit the totality of human rights in Europe. Otherwise, they risk to be forced away from Europe - and from humanity. Far from being a voyage of hope from barbarity to modernity, their journey from Africa to Europe turns into an archaic ordeal.

\section{KEYWORDS}

Queer Theory. Homonationalism. Leo Bersani. Lee Edelman. Jasbir Puar.

It is the duty of them that are in sovereign authority, to increase the people [...]. And seeing this is to be done by ordinances concerning copulation: they are by the law of nature bound to make such ordinances concerning the same, as may tend to the increase of mankind. And hence it cometh, that in them who have sovereign authority: not to forbid such copulations as are against the use of nature [...]. For though it be not evident, that a private man living under the law of natural reason only, doth break the same, by doing any of these things aforesaid; yet it is manifestly apparent, that being so prejudicial as they are to the improvement of mankind, that not to forbid the same, is against the law of natural reason, in him that hath taken into his hands any portion of mankind to improve.

(Thomas Hobbes)

\section{NEGATIVIDADE SEXUAL E SEUS BODES EXPIATÓRIOS}

Em 76 países do mundo a prática de relações sexuais consentidas entre pessoas do mesmo sexo é ilegal. Em sete desses países ${ }^{4}$ o culpado pode ser punido com pena de morte. Em alguns casos, apenas o sexo entre homens é criminalizado; em outros, homossexualidade não é mencionada em nenhum momento e pessoas não heterossexuais ou não conformadas com sua identidade de gênero são perseguidas por leis referentes a "atos sexuais contra a natureza”. Por outro lado, dos anos 2000 em diante o Alto Comissariado das Nações Unidas para Refugiados (UNHCR, 2002, 2008, 2012) passou a considerar a discriminação baseada em orientação sexual e identidade de gênero como violadoras dos direitos humanos. Consequentemente, pessoas lésbicas, gays, bissexuais, transgêneros e intersexuais (LGBTI), quando perseguidas em seus países de origem, deveriam ser aceitas como refugiados naqueles países que ratificaram a Convenção de Genebra. Dessa forma, todos os anos aproximadamente 10 mil pessoas LGBTI deixam sua terra natal para procurar asilo na Europa, seduzidas pela promessa dos direitos humanos que raramente é cumprida. Frequentemente uma

\footnotetext{
${ }^{4}$ Irã, estados do norte da Nigéria, Mauritânia, Arábia Saudita, partes do sul da Somália, Sudão e Iêmen.
} 
viagem de esperança, uma viagem em que frequentemente vidas são colocadas em risco, termina, de fato, em desilusões e desespero.

Empurrados ao subsolo quando estão em casa, muitas vezes perseguidos por seus pais e parentes, quando vêm da África são muitas vezes contrabandeados por traficantes como produtos ilegais juntamente com outros migrantes de quem ainda escondem sua sexualidade. Esmagados em contêineres como vacas eles cruzem os desertos, apertados em barcos improvisados eles partem. Alguns deles morrem durante a viagem, de sofrimento, tortura, ou em naufrágios. Aqueles que encontram mais sorte atingem a costa em centros de detenção para "imigrantes ilegais”, campos onde, novamente, são confrontados com discriminação, violência e abuso. Por fim, são “examinados” por comissões com pouco tempo a perder e não alheios a preconceitos e estereótipos, não raramente rejeitando seus pedidos. Em consequência, muitos dos requerentes de asilo retornam a seus países de origem, onde podem sofrer violência e tortura, ser presos ou sentenciados à morte. Aparentemente, em relação a pessoas LGBTI postulantes de asilo, os chamados “choques de civilização” (HUNTINGTON, 1996) acabam sendo a harmonia das civilizações.

Se nos dermos conta deste fracasso da cultura dos direitos humanos do Ocidente, primeiramente é necessário compreender quais são os “comportamentos” em questão, supostamente contra a natureza em 76 países do mundo; comportamentos esses que não são como quaisquer outros, que podem ser dominados por meio da vontade; ao que se dirige aqui não são as pessoas LGBTI e seus comportamentos indevidos, mas as motivações que os influenciam - na medida em que as motivações influenciam cada ser humano. Como Lee Edelman (2004; BERLANT; EDELMAN, 2014) reforça, precisamente por escapar ao controle individual, o sexual é considerado nas sociedades humanas algo excepcional, uma força inquietante que algumas categorias de pessoas, mais do que outras, são chamadas a personificar. Em outras palavras, esses comportamentos são identidades. Minorias sexuais não são perseguidas pelo que fazem, mas pelo que são: bodes expiatórios de uma negatividade que pertence a todos, os representantes da "bestialidade" da sexualidade humana, do que vai “contra a natureza” e, dentro da humanidade, ameaça seu futuro.

No esteio de Jean Laplanche (1992) e Leo Bersani (1987, 1996), Edelman diria que isso depende das estruturas de sexualidade inscritas em uma ordem simbólica, compreendida como um “anterior” a qualquer cultura. Não seria minha intenção endossar uma hipótese tão robusta. Irei restringir-me a algumas observações inicialmente derivadas não de leituras de teoria queer (BERNINI, 2013a, 2013b, 2014), mas de minhas experiências como um ativista queer e voluntário 
em associações italianas de apoio a requerentes de asilo LGBTI ${ }^{5}$. Então, terei como ponto de partida aquilo que sinto mais perto de mim, minha experiência como um cidadão gay da Itália e da Europa, no sentido de demonstrar que a negatividade queer se faz paradoxalmente presente não apenas onde pessoas LGBTI são perseguidas pela lei, mas também onde são protegidas pela lei. Minha intenção não é a de negar a necessidade de endossar e melhorar a cultura dos direitos humanos, mas de denunciar sua insuficiência e convidar a formas mais radicais de reflexão e ação.

\section{ENTRE PRAZER E TRADIÇÃO}

Como consequência da chamada "revolução sexual” o mundo ocidental se tornou uma sociedade de prazer (ŽIŽEK, 2008). O tempo em que o recato era considerado uma virtude pública, especialmente para mulheres, na verdade não se encontra muito distante; mas se assistirmos a um reality show ou a um talk show na televisão, ou simplesmente a propagandas, quando compararmos o que vemos com a experiência de nossas avós e bisavós teremos a sensação de termo-nos deslocado a uma nova era. Nos anos de 1970 Michel Foucault (1976) já havia apontado que a modernidade havia feito a sexualidade não mais o objeto de um silêncio, mas o objeto de um discurso, e Jacques Lacan (1978) que o capitalismo não é repressivo, mas transgressivo. Hodiernamente precisamos aproveitar, ter prazer, sem o que seremos considerados anormais, algo até mesmo patológico ${ }^{6}$. “Desfrutem!” o mercado diz ao consumidor. “Desfrutem!” o psiquiatra diz ao paciente. “Desfrutem!”, até mesmo os pais dizem a suas crianças. Hoje vivemos em uma sociedade do prazer em que o sexo, longe de representar um tabu, é considerado um direito, ou melhor, um dever que homens e mulheres devem a si mesmos.

Não obstante, “o sexual” continua um problema: se continuarmos a associá-lo a um imperativo (não importa se uma proibição ou uma obrigação), é porque tentamos exorcizar o fato de que é uma orientação perturbadora cujo poder não podemos evitar. No mesmo sentido, Bersani (1987,

\footnotetext{
${ }^{5}$ Em Milão fiz parte do projeto "Migrações e Homossexualidades” (Immigrazioni e Omossessualità) (disponível em: <https://goo.gl/Ue2z29>. Acesso em: 15 set. 2014), e em Verona no Centro de Apoio ao Migrante LGBT (Sportello migranti LGBT) (disponível em: <https://goo.gl/L3njct>. Acesso em 14 set. 2014).

${ }^{6}$ Desde os anos 2000, enquanto clamamos que para muitas pessoas a ausência de atração sexual é, de fato, uma orientação sexual, o movimento assexual denunciou como na cultura pós-moderna ocidental não é percebida como um objeto de proibição, mas como objeto de uma obrigação. A Rede de Visibilidade e Educação Assexual (Asexual Visibility and Education Network - AVEN) foi fundada em 2001 com o intuito de promover a despatologização e aceitação pública da sexualidade e para a facilitação do crescimento da comunidade assexual. Em seu website pode-se ler: "um assexual é alguém que não experimenta atração sexual. Diferentemente do celibato, em que pessoas escolhem, sexualidade é uma parte intrínseca do que somos. Assexualidade não faz nossa vida pior ou melhor, nós apenas enfrentamos uma gama de desafios diferentes do que a maioria das pessoas sexuais”. Disponível em: <https:/goo.gl/uvamkV>. Acesso em: 14 set. 2014.
} 
1996) afirma que seres humanos têm prazer no sexo, mas não gostam de sexo, visto que o sexual é uma ameaça à integridade do ego, ou melhor, à ilusão de que a essência dos egos humanos consiste em um domínio racional sobre impulsos e desejos. E tudo isso não se altera quando a disciplina da repressão é subvertida em uma celebração do prazer: o sexual continua uma inquietante, repugnante força que perturba nosso senso de humanidade, e nós precisamos de bodes expiatórios para representar sua negatividade; figuras de um pecado insustentável e campeões de uma excitante transgressão ao mesmo tempo.

Eu venho da Itália, um dos poucos países da Europa Ocidental que ainda não reconhece, de nenhuma forma, relações homossexuais e não defende minorias sexuais de crimes de ódio por meio de leis especificas ${ }^{7 ; 8}$. Mas mesmo em países em que leis protegem o casamento entre gays e lésbicas e a adoção por estes casais, assim como estabelecem a proteção de minorias sexuais, a homofobia, a transfobia e a bifobia não desapareceram completamente. De certa maneira, o consenso generalizado de que o completo reconhecimento legal de homossexualidade é equivalente a casamento, adoção e acesso a tecnologias reprodutivas para gays e lésbicas provam que o sexual ainda precisa ser salvo pelo afeto, amor e parentalidade para que seja aceito em nossa sociedade; que sexualidades não reprodutivas precisam se tornar reprodutivas para que possam ser limpas de sua negatividade; ou ainda, em outras palavras, que o sexual precisa se tornar algo diferente de si mesmo para que seja integrado como civilizado. “Desfrute!”, o mercado atualmente diz ao consumidor. “Desfrute!” diz o psiquiatra ao paciente. “Desfrute!” até mesmo os pais dizem aos filhos. Mas este imperativo é apenas uma dualidade obscena de uma lei moral, que permanece enraizada em tradições mesmo em sociedades ocidentais seculares, liberais e pós-modernas.

\section{QUEERCÍDIOS}

Uma das razões pela qual não deveríamos esquecer o quanto a cultura liberal contemporânea é ligada a tradições (e, portanto, conservadora) é porque a história dos direitos não possui um progresso liberal. Em Uganda, por exemplo, leis contra “atos sexuais não naturais” não existiam antes do colonialismo britânico do século XIX (HUMAN RIGHTS WATCH, 2008); ainda, as condições

\footnotetext{
${ }^{7}$ Ademais, a Itália possui uma legislação muito restritiva no que concerne à mudança de sexo para pessoas transgênero e não protege pessoas intersexuais de mutilações cirúrgicas (LORENZETTI, 2014).

${ }^{8}$ Sublinhamos que em 2016 o país aprovou lei que permitia a união civil entre casais homossexuais, todavia ainda permaneceu em $32^{\circ}$ lugar no ranking europeu de 49 países perigosos para a população LGBTI. A decisão de 2016 trouxe equidade em questões de impostos, segurança social e herança. In: International Lesbian, Gay, Bisexual, Trans and Intersex Association, 2016, State Sponsored Homophobia. Disponível em: <https://goo.gl/qu3VCU>. Acesso em: 6 jan. 2018. [N. do T.].
} 
das pessoas LGBTI pioraram muito nos últimos cinco anos desde que pastores evangélicos lançaram uma campanha anti-homossexualidade com o intuito de punir atos sexuais com pena de morte. A lei foi aprovada em dezembro de 2013, introduzindo a pena de prisão perpétua em vez de execução; também trouxe o banimento de todas as organizações que trabalhassem com direitos LGBTI. A Corte Constitucional de Uganda aboliu a lei em agosto de 2014, mas o debate ainda ocorre uma vez que a decisão se motivou apenas por irregularidades de procedimento no voto dos parlamentares. Outro exemplo é a Rússia, onde a homossexualidade foi descriminalizada em 1993, apesar de que em janeiro de 2013, com os aplausos de Vladimir Putin, nova lei tenha se estabelecido contra "propaganda homossexual” 9 . Poderíamos considerar que Uganda e Rússia encontram-se longe da Europa Ocidental e do suposto mundo civilizado ocidental - mas isso seria incidir em erro. Ambas as leis de Uganda e da Rússia foram, de fato, suportadas por organizações tradicionalistas dos Estados Unidos e da Europa (KAOMA, 2009, 2012), cuja campanha também em seus países de origem se orientava para a proibição de professores mencionarem discriminação sexual nas escolas com o intuito de prevenir bullying. Recentemente, testemunhamos claramente instâncias deste movimento transnacional na França e na Itália; e não podemos nos olvidar que em 1988 a Inglaterra de Thatcher proibiu autoridades locais de "promoverem" a homossexualidade ${ }^{10}$.

Em 26 de janeiro de 2011, aos 46 anos, David Kato Kinsule foi violentado em sua casa em Bakusa, Uganda, sendo agredido com marteladas na cabeça até a morte: ele era um dos fundadores do movimento Minorias Sexuais de Uganda e havia recentemente vencido uma batalha judicial contra o tabloide local Rolling Stones, que fazia uma chamada pública com seu nome e rosto, pedindo seu enforcamento. Mas queercídios não são apenas um problema africano. Em seis de outubro de 1998, em Wyoming, Estados Unidos, o estudante de 22 anos Matthew Shepard foi espancado, agredido com uma pistola e torturado. Ele foi encontrado amarrado em uma cerca, em coma, dezoito horas depois. Seis dias mais tarde ele faleceu no hospital. Sua culpa era ser gay. Os assassinatos de Kato e Shepard foram condenados pela mídia e pela sociedade civil, mas poucas pessoas no mundo reagem quando uma pessoa transgênero morre: mais de 1.500 são as mortes desse tipo reportadas de janeiro

\footnotetext{
${ }^{9}$ A nova lei russa é contra a circulação de qualquer informação sobre a existência de não heterossexuais e de subjetividades de gênero não conformes.

${ }^{10}$ Seção 28 do Local Government Act, promulgado em 1988. No Reino Unido, relações sexuais entre pessoas do mesmo sexo foram descriminalizadas em 1967.
} 
de 2008 a março de $2014^{11}$, 98 delas na América do Norte e 87 na Europa - onde a Itália é a segunda colocada $^{12}$, perdendo apenas para a Turquia ${ }^{13}$.

\section{O NÓ HOBBESIANO}

Como assevera Jasbir Puar (2007), os Estados Unidos e a Europa não deveriam se sentir muito confortáveis celebrando o secularismo e o liberalismo de suas sociedades e condenando o atraso dos países do eixo sul, orientais e islâmicos. Ainda, seus governos não deveriam se utilizar dos avanços em termos dos direitos civis LGBTI nas sociedades ocidentais como pretexto para justificar seu imperialismo e ganhar apoio juntamente ao público liberal. Entretanto - no ritmo de Puar e suas críticas ao homonacionalismo ${ }^{14}$ e ao pinkwashing ${ }^{15}$ - quem pertence a este público liberal, eu incluído, não pode deixar de celebrar o fato de que pessoas LGBTI, quando perseguidas em seu país de origem, podem reclamar asilo em países que ratificaram a Convenção de Genebra. Isso abre, todavia, uma série de novas questões. Como, em que medida, e a que preço os representantes de uma força que incomoda nosso senso de humanidade, como o sexual ainda permanece, podem se beneficiar dos direitos humanos?

Da perseguição dos judeus pelo regime nazista (ARENDT, 1958; BUTLER; SPIVAK, 2007) até o recente debate sobre o projeto de lei anti-homossexualidade em Uganda, evidências históricas demonstram que não há nada "natural” nos direitos humanos, nada neles procede diretamente da natureza humana. Isso fica particularmente claro em algumas passagens do filósofo britânico Thomas Hobbes (1640, 1642, 1651), que é considerado universalmente como o fundador do pensamento político moderno e cujo trabalho é considerado fundamental para a compreensão do conceito de biopolítica cunhado por Michel Foucault (1976, 1997, 2004a, 2004b) e desenvolvido por Giorgio Agamben (1995). De acordo com Hobbes, estar incluído na humanidade depende de uma decisão, a qual estaria vinculada apenas a um poder soberano (SCHMITT, 1963). Como fonte de direitos, para Hobbes, a natureza humana em si é uma convenção social, ou ainda um conceito político. Em caso de dúvidas, apenas o soberano teria o poder para definir este conceito, decidindo o que é natural e o

\footnotetext{
${ }^{11}$ Fonte: Transgender Europe, Trans Murder Monitoring Project, abril de 2014. Disponível em: <https://goo.gl/hYzZ8j>. Acesso em: 15 set. 2015.

1227 homicídios reportados.

1335 homicídios reportados.

${ }^{14}$ Homonacionalismo é a forma de patriotismo ocidental baseado na ideia da excepcionalidade do mundo ocidental em promover os direitos das minorias sexuais.

${ }^{15}$ Pinkwashing é a estratégia que pretende justificar o imperialismo ocidental como uma desculpa para liberar mulheres e minorias sexuais da opressão que sofrem em países não ocidentais.
} 
que não é natural para o humano, quem completamente pertence à natureza humana e quem deve ser considerado menos que humano, quem merece e quem não merece a proteção dos direitos humanos (BERNINI, 2013b) ${ }^{16}$.

Os 76 países mencionados no começo deste texto deixaram clara a escolha de excluir a população LGBTI da sua ideia de humanidade. Não tão claras, todavia, são as escolhas feitas por países europeus, apesar das recomendações do Alto Comissariado das Nações Unidas para Refugiados. Seguindo a chamada Regulamentação de Dublin a respeito de imigração, em cada país europeu foram instituídas comissões territoriais com o intuito de examinar pedidos de asilo; mas pesquisas recentes (JANSEN; SPIJKERBOER, 2011; SPIJKERBOER, 2013) revelam que requerimentos de pessoas LGBTI têm sido regularmente rejeitados ${ }^{17}$. Em diversos casos, comissões alegam ser implausível que os requerentes de asilo tenham tomado parte em atos tão ameaçadores a suas vidas. Em outros casos, a motivação é a de que aqueles que pertencem a uma minoria sexual podem evitar a perseguição “apenas” por esconder sua identidade. Então ocorre que bissexuais, "lésbicas que não se comportam de maneira masculina, gays não afeminados”, e requerentes que “tenham sido casados ou tenham filhos” (JANSEN; SPIJKERBOER, 2011, p. 7), raras vezes obtêm asilo: aparentemente para as comissões essas pessoas devem preferir estar em seus países de origem,

\footnotetext{
${ }^{16}$ Hobbes (1640, capítulo XXVIII) aponta, por exemplo, que o soberano tem o dever de aprimorar e incrementar a vida das pessoas que governa, e, portanto, proibir “coitos que sejam contra o uso da natureza”, apesar de não ser evidente que um indivíduo em estado de natureza iria contrariar alguma lei da natureza por tomar parte nesses coitos. E ele ainda assevera (1642, capítulo XVII) que em caso de dúvidas apenas a lei - que seria a vontade do soberano - poderia, em última instância, decidir se um nascituro "de forma insólita” teria que ser considerado humano, e consequentemente protegido contra homicídio. Para a sensibilidade contemporânea, Hobbes pode soar um tanto cruel, e o é. Mas ele talvez soe menos espantoso se considerarmos que em países seculares é designada ao parlamento, que é soberano, a decisão de quais tipos de atos sexuais e em que idade devem ser considerados legais, bem como sob quais condições aborto e eutanásia não devem ser considerados homicídios.

${ }^{17}$ A Convenção de Dublin foi assinada em 1990 e em 2003 foi substituída pela II Regulamentação de Dublin (n. 343/2003). Recentemente, em 2013, a III Regulamentação de Dublin (n. 604/2013) entrou em vigor, introduzindo uma inovação fundamental: nenhum requerente de asilo poderia, a partir de então, ser transferido a um Estado membro onde corresse risco de ser sujeitado a tratamentos desumanos ou degradantes. Outro importante passo dado na recepção de requerentes de asilo LGBTI é representado pela Diretiva 2013/32 Dos Procedimentos Comuns para Concessão e Rescisão de Proteção Internacional. O artigo 24 estabelece de fato que “certos requerentes podem necessitar de garantias procedimentais especiais devido a, entre outros, idade, gênero, orientação sexual, identidade de gênero, deficiência, doença grave, transtorno mental ou sob consequência de tortura, estupro ou outras formas graves de violência física, psicológica ou sexual. Os Estados membros devem se esforçar em identificar os requerentes que necessitam de procedimento especial e garantias antes de uma decisão de primeira instância. Os requerentes devem receber tratamento adequado, incluindo tempo suficiente, no sentido de criar as condições necessárias para seu efetivo acesso aos procedimentos e para a apresentação dos elementos requeridos para substanciar seus pedidos de proteção internacional” [Tradução livre do seguinte texto oficial: “Certain applicants may be in need of special procedural guarantees due, inter alia, to their age, gender, sexual orientation, gender identity, disability, serious illness, mental disorders or as a consequence of torture, rape or other serious forms of psychological, physical or sexual violence. Member States should endeavour to identify applicants in need of special procedural guarantees before a first instance decision is taken. Those applicants should be provided with adequate support, including sufficient time, in order to create the conditions necessary for their effective access to procedures and for presenting the elements needed to substantiate their application for international protection”].
} 
dentro do armário, fingindo ser heterossexuais, a tentar escapar de perseguições. Gays e lésbicas que correspondem a estereótipos - digamos, gays “espalhafatosos” e lésbicas “caminhoneiras” - devem, portanto, ter mais chances de obter proteção internacional: mas paradoxalmente mulheres trans ou de gênero fluido cuja condição é patente tem regularmente sua proteção negada porque os comissionários atribuem a violência que sofrem mais à prostituição do que à sua identidade de gênero.

\section{O TORMENTO DA HUMANIDADE}

Aparentemente, mesmo nos países em que gays e lésbicas tenham obtido pleno acesso à cidadania como maridos, esposas, pais e mães, a população LGBTI requerente de asilo tem enfrentado graves dificuldades em seu reconhecimento como merecedores de proteção dos direitos humanos enquanto pessoas LGBTI. Sua urgência em viver de acordo com seus sentimentos e desejos não é geralmente considerada um direito fundamental derivado da natureza humana. Leis europeias delegaram a comissões territoriais o poder soberano de decretar às fronteiras de ambos a humanidade e as “adequadas subjetividades LGBTI”. Raramente este poder é usado para reconhecer a força incômoda do sexual como naturalmente humano: ainda identificado como os bodes expiatórios da negatividade sexual, requerentes de asilo LGBT são regularmente retornados para sua terra natal. Como consequência, longe de ser uma viagem de esperança, da barbaridade para a modernidade, suas jornadas da África para a Europa acabam sendo um arcaico tormento. Banidos da humanidade não pelo que fizeram, mas pelo que são, eles arriscam suas vidas para alcançar a Europa por terra e mar. Mas por uma reviravolta do destino, aterrissam nas mesmas ilhas em que o Fascismo italiano costumava confinar homens homossexuais (BENADUSI, 2005; GORETTI; GIARTOSIO, 2006; BERNINI, 2013c). Aqui são presos, colocados em campos e subjugados a seu último julgamento: apenas se conseguirem convencer uma comissão soberana de seu direito de ser quem são eles irão se beneficiar da totalidade dos direitos humanos na Europa. Caso contrário, arriscam-se a serem forçados a se retirar da Europa - e da humanidade novamente.

Ante essa tragédia, a resposta liberal que qualquer cidadão liberal de um país democrático deveria endossar é a demanda de direitos humanos para pessoas LGBTI enquanto pessoas LGBTI, seu direito a serem universalmente e definitivamente recebidas dentro das fronteiras da humanidade. Isso é o que Judith Butler (2000, 2004), por exemplo, postula. Mas uma reação diferente, e não necessariamente em contradição com a primeira, é possível e, ainda, sugerida por outros teóricos queer como Edelman (2004) e Puar (2007). O fato de que nos Estados Unidos e na Europa o pleno reconhecimento à homossexualidade deve passar por casamento e parentalidade prova que em nossas 
sociedades o desfrute do sexual ainda deve se render ao amor para ser socialmente aceito. No século XXI, seres humanos continuam desfrutando o sexo em países ocidentais, mesmo sem gostar de sexo (BERSANI, 1987, 1996), e a lei ainda aparenta não ser completamente capaz de render essas pessoas que são feitas para incorporar a inquietante força do sexual de sua negatividade social. Mais que clamar que essa força seja reconhecida como propriamente humana, uma reação queer diferente poderia ser buscar a coragem de aceitar pessoas LGBTI como alheias à humanidade para não apenas apostar em seus clamores pelos direitos do não humano ou do pós-humano (BRAIDOTTI, 2013), mas para atrapalhar a lógica biopolítica da soberania e dos direitos na totalidade.

O Parlamento de Uganda, Vladimir Putin e os movimentos transnacionais reacionários antiLGBTI podem, então, estar corretos em temer os possíveis efeitos das campanhas educacionais para respeito das minorias sexuais. Algumas formas de “propaganda queer” podem, de fato, acabar sendo muito perigosas (EDELMAN, a ser publicado). Elas poderiam desafiar o poder das autoridades soberanas; e mesmo ultrapassar a ideia ocidental de civilização (HOCQUENGHEM, 1972).

\section{REFERÊNCIAS}

Agamben, G. (1995), Homo sacer, Torino, Einaudi.

Arendt, H. (1958), The Decline of the Nation-State and the End of the Rights of Man, in The Origins of Totalitarianism, San Diego, New York and London, Harcourt Brace \& Company.

Benadusi, L. (2005), Il nemico dell'uomo nuovo: L'omosessualità nell'esperimento totalitario fascista, Feltrinelli, Milano.

Berlant, L. and Edelman, L. (2014), Sex, or the Unbearable, Durham and London, Duke University Press.

Bernini, L. (2013a), “Nessuna pietà per il piccolo Tim: Hocquenghem, Edelman e la questione del futuro", in «AG AboutGender: Rivista internazionale di studi di genere», Vol. 2, Nº 3.

Bernini, L. (2013b), Apocalissi queer: Elementi di teoria antisociale, Pisa, Edizioni ETS.

Bernini, L. (2013c), Luoghi di confino, linee di confine: Per un'ontologia anarchica dell'umano, in C. Rinaldi (ed.), La violenza normalizzata: Omofobie e transfobie negli scenari contemporanei, Torino, Kaplan.

Bernini, L. (2014), “The 'Post' in The Past: Queer Radicalism - in the Spirit of Stonewall”, in «AG AboutGender: Rivista internazionale di studi di genere», Vol. 3, № 6 .

Bersani, L. (1996), Homos, Cambridge (Massachusetts), Harvard University Press. 
Braidotti, R. (2013), The Posthuman, Cambridge, Polity Press.

Bersani, L. (1987) Is the Rectum a Grave?, in Id., Is the Rectum a Grave? And Other Essays (2010), Chicago, University of Chicago Press.

Butler, J. (2000), Antigone's Claim: Kinship between Life and Death, New York, Columbia University Press.

Butler, J. (2004), Undoing Gender, New York and London, Routledge.

Butler, J. and Spivak, G. C. (2007), Who Sings the Nation State? Language, Politics, Belonging, New York and Oxford, Seagull Books 187.

Edelman, L. (2004), No Future: Queer Theory and the Death Drive, Durham and London, Duke University Press.

Edelman, L. (forthcoming), Bad Education, Durham and London, Duke University Press.

Foucault, M. (1976), La volonté de savoir, Paris, Gallimard.

Foucault, M. (1997), “Il faut défendre la societé”: Cours au Collège de France, 1975-1976, Paris, Seuil-Gallimard.

Foucault, M. (2004a), “Sécurité, territoire, population”: Cours au Collège de France, 1977-1978, Paris, Seuil-Gallimard.

Foucault, M. (2004b), “Naissance de la biopolitique”: Cours au Collège de France, 1978-1979, Paris, Seuil-Gallimard.

Goretti, G. and Giartosio, T. (2006), La città e l'isola: Omosessuali al confino nell'Italia fascista, Donzelli, Roma.

Hobbes, T. (1640), Elements of Law Natural and Politic, 1969 ed. by F. Tönnies, London, Frank Cass \& Co.

Hobbes, T. (1642), De cive, 1984 ed. by H. Warrender, Clarendon Edition of the Works of Thomas Hobbes, Oxford, Clarendon Press.

Hobbes, T. (1651), Leviathan, or the Matter, Forme, \& Power of a Common-Wealth Ecclesiastical and Civil, 2007 ed. by R. Tuck, Cambridge, Cambridge University Press.

Hocquenghem, G. (1972), Le Désir homosexuel, Paris, Éditions universitaires.

Human Rights Watch (2008), This Alien Legacy: The Origin of "Sodomy" Laws in Bristish Colonialism, New York, Human Rights Watch.

Huntington, S.P. (1996), The Clash of Civilizations and the Remaking of World Order, New York, Simon \& Schuster. 
Jansen S. and Spijkerboer, T. (eds. 2011), Fleeing Homophobia: Asylum Claims Related to Sexual Orientation and Gender Identity in Europe, COC Nederland - Vrije Universiteit Amsterdam.

Kaoma, K.J. (2009), Globalizing the Culture Wars: U.S. Conservatives, African Churches, \& Homophobia, Somerville, Political Research Associates 188.

Kaoma, K.J. (2012), Colonizing African Values: How the U.S. Christian Right is Transforming Sexual Politics in Africa, Somerville, Political Research Associates.

Lacan, J. (1978), Du discours psychanalytique, in G. Contri (ed.), Lacan in Italia/Lacan en Italie (1953-1978), Milano, La Salamandra.

Laplanche, J. (1992), La révolution copernicienne inachevée (Travaux 1967-1992), Paris, Aubier.

Lorenzetti, A. (2014), Diritti in transito: La condizione giuridica delle persone transessuali, FrancoAngeli, Milano.

Puar, J.K. (2007), Terrorist Assemblages: Homonationalism in Queer Times, Durham and London, Duke University Press.

Schmitt, C. (1963), Der Begriff des Politischen, München-Leipzig, Dunker \& Humblot Spijkerboer, Th. (ed., 2013), Fleeing Homophobia: Sexual Orientation, Gender Identity and Asylum, Routledge, London and New York.

UNHCR (2002, 7 May), Guidelines on International Protection No. 1: Gender-Related Persecution Within the Context of Article $1^{\circ}(2)$ of the 1951 Convention and/or its 1967 Protocol Relating to the Status of Refugees.

UNHCR (2008, 21 November), Guidance Note on Refugee Claims Relating to Sexual Orientation and Gender Identity.

UNHCR (2012, 23 October), Guidelines on International Protection No. 9: Claims to Refugee Status based on Sexual Orientation and/or Gender Identity within the context of Article 1A(2) of the 1951 Convention and/or its 1967 Protocol relating to the Status of Refugees.

Žižek, S. (2008), For They Know not What They Do: Enjoyment as a Political Factor, London and New York, Verso. 